\title{
In Memoriam: Jane Smiley Cronin Scanlon
}

\section{Edward F. Aboufadel}

Jane Smiley Cronin Scanlon passed away on June 19, 2018, at the age of ninety-five. She was one of the most prominent female mathematicians of the twentieth century, known for her professional contributions to functional analysis, differential equations, and mathematical biology. During her career, Scanlon published more than fifty papers, as well as two research monographs [Cr2, Cr4] and two textbooks [Cr3, CS1]. In this remembrance, we will review highlights from her remarkable life and career, particularly her work at Rutgers University. Scanlon was a member of the AMS since 1942.

\section{Early Career}

Scanlon ${ }^{1}$ was born in Manhattan and attended high school in Highland Park, Michigan. After graduating from Wayne State University with a BS in mathematics, she completed her dissertation in 1949 at the University of Michigan on the topic "Branch Points of Solutions of Equations in Banach Space," supervised by Erich Rőthe. The thesis dealt with nonlinear integral equations connected to results of Schmidt and Liapounoff. It was published in the Transactions of the American Mathematical Society in 1950 [Cr1].

Before joining the faculty of Polytechnic Institute of Brooklyn $^{2}$ in 1957, Scanlon held postdoctoral fellowships

Edward F. Aboufadel is an associate vice president for academic affairs and professor of mathematics at Grand Valley State University. His email address is aboufade@gvsu.edu.

Communicated by Notices associate editor Reza Malek-Madani.

${ }^{1}$ Jane Smiley Cronin married physicist Joseph Scanlon in 1953, after she had established her publishing career. She continued to publish primarily under her maiden name, but from 1967 to 1977 she had eleven publications as Cronin-Scanlon. The two divorced in 1979, although she continued to use the Scanlon name professionally at Rutgers [Mu].

${ }^{2}$ A predecessor of the New York University Tandon School of Engineering. For permission to reprint this article, please contact: reprint-permission @ams.org.

DOI: https://dx.doi.org/10.1090/noti 1948

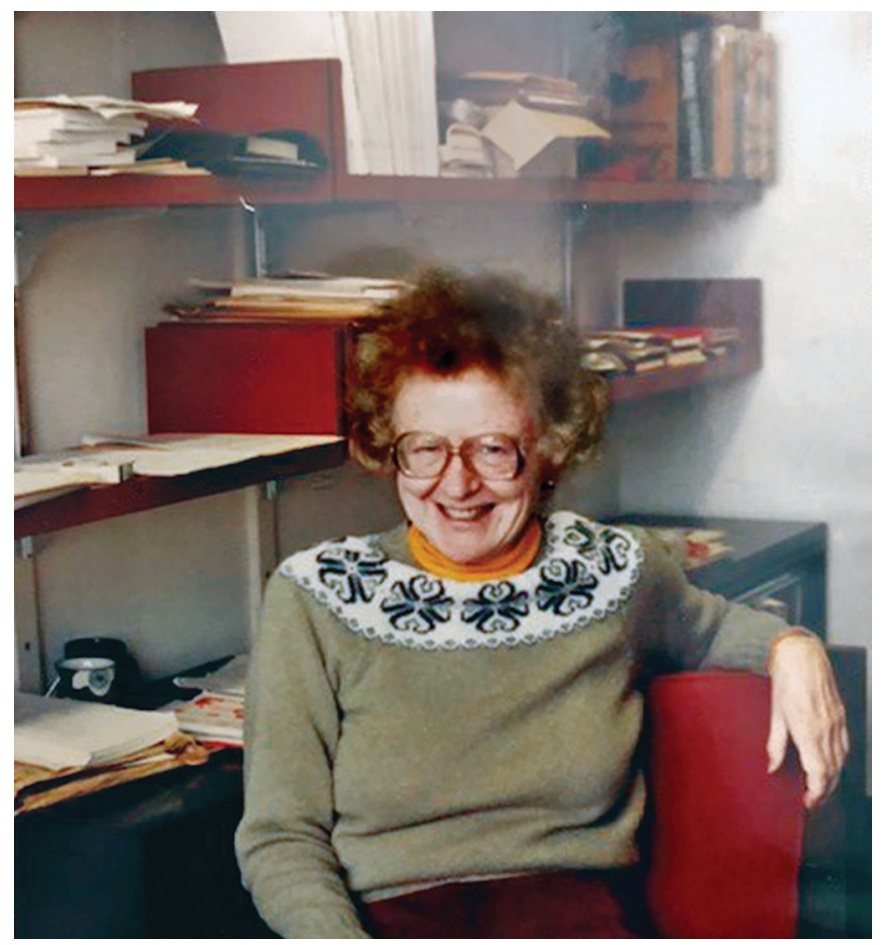

Enjoying a moment in her office at Rutgers University, 1989.

at Princeton University, the University of Michigan, and the Courant Institute of Mathematical Sciences; worked as a mathematician at the Air Force Cambridge Research Center and the American Optical Company; and taught at Wheaton College and Stonehill College, both in Massachusetts. In those early years, Scanlon published several results in the area of functional analysis and received grants from the Office of Naval Research and the National Science Foundation $[\mathrm{Mu}]$.

From 1957 to 1965 Scanlon turned her focus to differential equations, particularly the search for periodic and almost-periodic solutions. One outstanding accomplishment during that time was the publication in 1964 of her 
critically acclaimed monograph Fixed Points and Topological Degree in Nonlinear Analysis by the AMS [Cr2]. In Mathematical Reviews, Solomon Lefschetz of Princeton, an early supporter of Scanlon [CS2], began his commentary on this work as follows: "This very interesting monograph contains a systematic treatment of fixed-point and local degree theory as it applies to differential equations and related topics" [Le]. Focusing on Poincaré's bifurcation equation for the quasi-linear $n$-vector system:

$$
d x / d t=A(t) \mathbf{x}+\mu f(x, t, \mu)+g(t)
$$

where $A$ is an $n$-by- $n$ matrix, $\mu$ is a small, real parameter, and the functions of $t$ have period $T$, Scanlon determined properties to guarantee resonance and expanded the analysis to almost-periodic solutions. This was followed in the monograph by a chapter on fixed-point and degree theorems from topology, which set up applications to certain integral and elliptic partial differential equations.

The 1964 monograph was published less than thirty years after the influential paper of Leray and Schauder [LS], in which they generalized finite-dimensional topological degree to an important class of maps on Banach spaces and showed the usefulness of this extension to problems in analysis. The monograph was important in that it brought results on topological degree, fixed-point theory, and applications to a broader mathematical and scientific audience, and it set the stage for the next phase of Scanlon's career.

\section{Advancing on New Brunswick}

During the 1960s, Rutgers University began an initiative to hire strong, research-active women into the mathematics department. Based on her substantial publication record, Scanlon was recruited to join Rutgers in 1965. This advancement of the department was led by Ken Wolfson, who served as chair from 1961 to 1975 [Mu]. Some details about this initiative are available on the website for the history of the Rutgers mathematics department ${ }^{3}$ [We1]:

[By the late 1960s] Rutgers had practically cornered the market on women mathematicians ... . In addition to Katharine Hazard (at Douglass) and Barbara Osofsky, the various math departments had now acquired Helen Nickerson (1960), Jacqueline Lewis (1963, University College), Joanne Elliott (1964), Jane Scanlon (1965), and Patricia Tulley McAuley (1965, Douglass). Thus 7 of the 32 senior fac-

\footnotetext{
${ }^{3}$ At the time, Rutgers University's main campus in New Brunswick had three distinct mathematics departments until the 1980 merger: Douglass (the women's college), University College (for returning adults), and Rutgers College (a men's college until 1972). Scanlon was appointed to Rutgers College.
}

ulty $(22 \%)$ were women. The national average was under $1 \%$ women then.

The initiative to hire distinguished women mathematicians continued into the 1970s with the hiring of Tilla Klotz Milnor Weinstein, Jean Taylor, and Amy Cohen. Weinstein chaired the Douglass department from 1970 to 1973 and from 1977 to 1980 [Mu, We2].

By the end of her first decade at Rutgers, Scanlon's research interests had evolved, with a new focus on mathematical biology. Specifically, she concentrated on qualitative analysis of systems of differential equations that modeled dynamics seen in various biological systems. Scanlon was particularly interested in cell electrophysiology described in cardiac models (e.g., Purkinje fibers) [Cr5] and in the Hodgkin-Huxley model of the giant nerve fiber in the squid [GHW]. She also studied equations that arise in the study of catatonic schizophrenia [CS3]. Scanlon's turn to applied mathematics fit in with an initiative in the early 1970s to strengthen the applied mathematics program at Rutgers.

\section{Qualitative Analysis: From van der Pol to Hodgkin-Huxley}

In 1987 Scanlon published her second research monograph on the Hodgkin-Huxley system of differential equations [Cr4]. The van der Pol system of differential equations provides an accessible introduction to some of the ideas discussed in her book.

Developed in the early twentieth century to model the electrical current through a specific circuit that possesses stable oscillations, the second-order differential equation

$$
d^{2} x / d t^{2}+\mu\left(x^{2}-1\right) d x / d t+x=0
$$

was derived by van der Pol. Here, $\mu$ is a large parameter that represents the strength of the nonlinear damping. Rescaling the time variable $t$ by dividing by $\mu$ and introducing

$$
y=\varepsilon d x / d t+x^{3} / 3-x
$$

with the new $t$, along with $\varepsilon=1 / \mu^{2}$, a very small, positive parameter, one obtains this system of equations:

$$
d x / d t=(1 / \varepsilon)\left(x-x^{3} / 3+y\right), \quad d y / d t=-x .
$$

We refer to this as a singularly perturbed system, owing to the presence of $1 / \varepsilon$. It has a periodic solution that is called a relaxation oscillation.

There are a number of ways to study relaxation oscillations, and one of Scanlon's primary interests was the qualitative analysis approach. The basic idea is to consider the direction of solutions to (4) at various points in the plane. Because $\varepsilon$ is very small (representing large damping), 


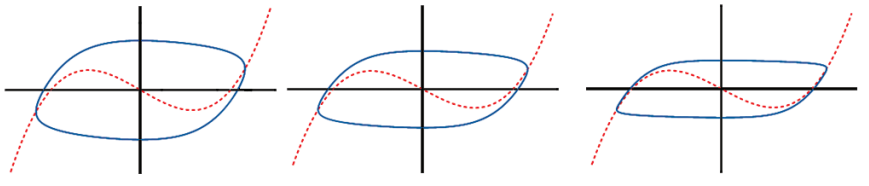

Figure 1. van der Pol relaxation oscillations (clockwise trajectories) for $\varepsilon=0.5,0.3$, and 0.1. The dashed curve is the slow manifold.

we focus on the "s-curve" $y=x^{3} / 3-x$, which is called the slow manifold. Nonstationary solutions to (4) have vertical tangents when they intersect this curve.

On the other hand, for points to the left of the slow manifold, we have $y>x^{3} / 3-x$, so $d x / d t$ is positive and relatively large, while $d y / d t$ is relatively small in absolute value, due to the scaling difference of $\varepsilon$. A similar statement can be made to the right of the slow manifold. Consequently, solutions are attracted to the two tails of the slow manifold and move there quickly, in what is sometimes called the fast subsystem. Near the slow manifold, solutions are nearly vertical and move slowly (hence the term slow manifold). Overall, the solution will spiral in a sequence of fast and slow (or relaxing) pieces, converging to the periodic relaxation oscillation that exists for the van der Pol equations (4). See Figure 1 and [GHW]. To prove the existence of the oscillation, an attracting annulus is defined in the plane, and fixed-point theory is applied (see Figure 2).

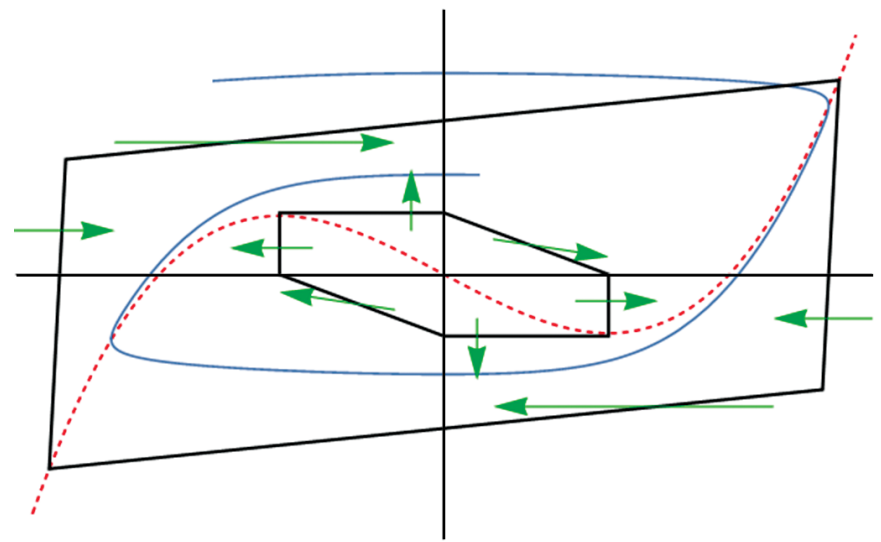

Figure 2. An example of an attracting annulus for the van der Pol autonomous system, with inflow trajectories indicated, as well as a sample solution for $\varepsilon=0.1$ that enters and is trapped in the annulus.

The idea of fast and slow pieces can also be applied to the Hodgkin-Huxley equations. Alan Hodgkin and Andrew Huxley, along with Sir John Eccles, were jointly awarded the Nobel Prize in Physiology or Medicine in 1963 "for their discoveries concerning the ionic mechanisms involved in excitation and inhibition in the peripheral and central portions of the nerve cell membrane," including what is now known as the Hodgkin-Huxley system of differential equations [No]. The Hodgkin-Huxley model consists of four differential equations capturing the activation of potassium and sodium gates in the giant axon cells of a squid [ET]. One of the four equations, for the membrane potential $\mathrm{V}$, has a similar structure to the $d x / d t$ equation in (4) in that there is a small membrane capacitance variable $c_{M}$ such that

$$
d V / d t=\left(1 / c_{M}\right) F(V, h, m, n),
$$

where $h, m$, and $n$ are the three other dependent variables in the system. Similar to the qualitative analysis of the van der Pol oscillator, $F(V, h, m, n)=0$ is the slow manifold, an "s-shaped" curve in four-dimensional space, while the other variables are associated with fast-relaxing dynamics. However, the technique of creating an attracting annulus does not apply.

Scanlon's monograph [Cr4] on the Hodgkin-Huxley differential equations is described as "a delightful book" [Sl] and features the derivation of the equations from physiological ideas, various modifications and simplifications of the equations, the modeling of muscle and the cardiac Purkinje fibers, and both qualitative and quantitative analysis of the system, including singular perturbations. Scanlon was also interested in entrainment of frequency problems, where equations like (2) had an additional forcing term with a different period than the underlying relaxation oscillation, exploring the question of whether a periodic solution exists with the period of the forcing term. Scanlon's analyses of these problems, utilizing a variety of analytic tools, were published in 1996 [Cr6].

\section{Celebrating an Outstanding Woman Mathematician}

In the latter part of her career, Scanlon was recognized for her influential work in pure and applied mathematics. She spent the 1984-85 academic year at Courant, funded via a Visiting Professorship for Women from the National Science Foundation. In 1985 she was the Emmy Noether Lecturer for the Association for Women in Mathematics. In that lecture she described the model of the Purkinje fiber and a qualitative analysis to prove the existence of periodic solutions. At the AMS/MAA joint meetings in 1989, Scanlon gave the talk "Entrainment of frequency" for the Pi Mu Epsilon Sutherland Frame Lecture.

Scanlon was an Emeritus Member of the AMS, due to her being a member for at least fifty years. She also was an active member. Along with being a regular speaker at sectional and national AMS meetings, she served on the AMS Council (1974-76), the Nominating Committee (1980-81, chair in 1980), the Gibbs Lecturer Committee (1988-89), and the Steele Prize Committee (1992-94), as well as the Short Course Subcommittee of the Program Committee for National Meetings and the Proceedings of Symposia in Applied Mathematics Editorial Committee. In 1998 Scanlon and Robert O'Malley organized an AMS Short 
Course, Analyzing Multiscale Phenomena Using Singular Perturbation Methods, in Baltimore [CM].

Scanlon was also an important figure in raising the visibility of women in mathematics in the twentieth century. She was a founding member in 1972 of the AMS Committee on Women in Mathematics. For the 822nd meeting of the AMS in Amherst, Massachusetts, in 1985, she organized and spoke at the session Sonya Kovalevskaya: Major currents in 19th century mathematics [Bl]. She was also featured in Margaret Murray's 2000 book Women Becoming Mathematicians: Creating a Professional Identity in Post-World War II America [Mu].

Through interviews with Scanlon, Murray sheds light on many aspects of Scanlon's career. For instance, Scanlon's decision to choose to major in mathematics rather than physics at Wayne State University is described in this way: "In short, mathematics offered a clarity that no empirical science could match." In an extensive discussion of Scanlon's time in graduate school at Michigan, Murray shares details on Scanlon's opinion of the teaching styles of a number of professors, the anxiety of being a graduate student, and the exhilaration of discovering research in collaboration with her advisor, Erich Röthe. In her postdoctoral positions, Scanlon "developed the habit of research and publication" that was critical to her success in the 1950s and 1960s. In summarizing her career, Murray writes that "Jane Cronin Scanlon presented herself as a professional to be reckoned with on her own terms."

In 1991 the AMS published a special issue of the Notices on women in mathematics. That issue includes a history of the AWM where Lenore Blum described the challenges of being a woman studying mathematics in graduate school in the 1970s and not being aware of other women mathematicians [Bl]. Blum included Scanlon in the list of "the strong women contemporaries who could very well have served as important role models and mentors had we known of their existence early on: Mina Rees, Julia Robinson, Mary Ellen Rudin, Cathleen Morawetz, Olga Taussky-Todd, Jane Cronin Scanlon, and Marian Pour-El."

In the wake of her passing, Scanlon's colleagues spoke highly of her time at Rutgers. Fred Roberts observed that her marriage of pure and applied mathematics was a good fit for the department and set the stage for the creation of DIMACS (Center for Discrete Mathematics and Theoretical Computer Science) in 1989. After it was established, Scanlon was particularly welcoming of visitors to the new center. Joseph McDonough, one of her graduate students, notes that "Jane was a very down-to-earth person who never lost her sense of wonder." Amy Cohen recalled that Scanlon "took the notion of the three-corner stool seriously-teaching, research, and service" and that Scanlon promoted the idea of "a community of scholars rather than a competition of scholars."

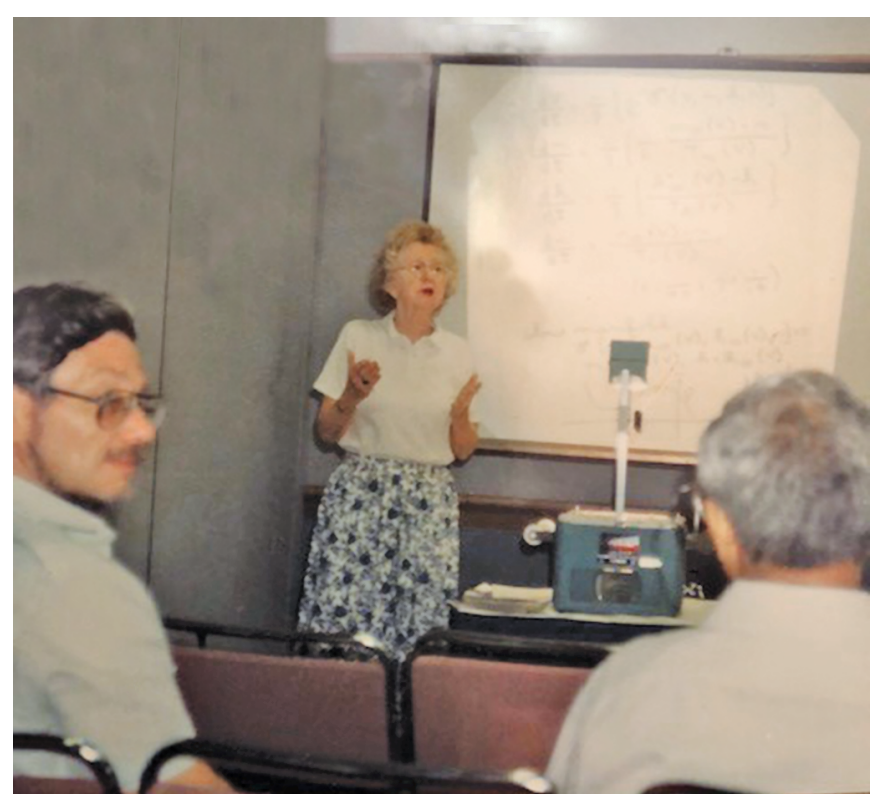

Seminar time at Rutgers, 1988.

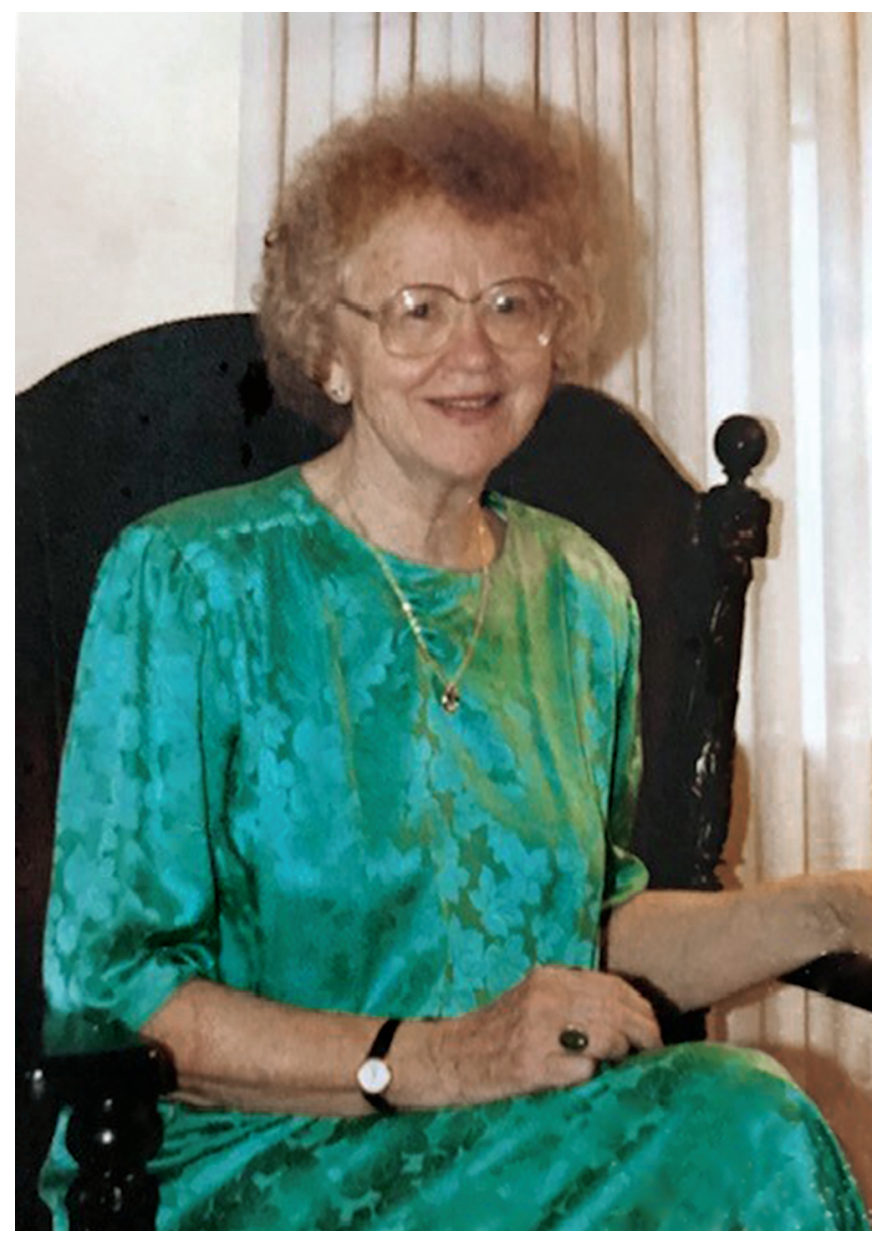

Ready to celebrate Christmas, 1992. 
Scanlon also had broad nonmathematical interests, in particular, fencing, literature, and genealogy. Regarding the intersection of mathematics and politics, her son, Justin Scanlon, said that "she taught me very early that one of the most overlooked aspects of studying history is how people count things. For example, how do we determine the number of people who die in a war?" The way we count is critical to the way we understand politics and history.

In a similar vein, her daughter, Anne Yamakaitis, noted that "she was not one to allow for shallow thought," providing the following anecdote from Scanlon's last year of life:

While recovering from surgery, a therapist asked her why she daily read both the New York Times and The Wall Street Journal. My mother, somewhat incredulous that such a silly question could even be raised, pointed out the obvious: that in reading only one or the other the reader would gather biased information of economic or world news.

During Jane Scanlon's twenty-six years at Rutgers University, seven students completed PhDs under her: Clifton Lando (1969), Douglas McCarthy (1970), Max Ashkenazi (1978), Stephen Hoyle (1978), Joseph McDonough (1979), Neil Greenbaum (1985), and the author of this article (1992).

A star in the constellation of twentieth-century female mathematicians, Jane Smiley Cronin Scanlon became professor emerita in 1991.

\section{References}

[Bl] Blum L. A Brief History of the Association for Women in Mathematics: The Presidents' Perspectives. Notices Amer. Math. Soc. (38): 738-754, 1991. MR1125380

[Cr1] Cronin J. Branch points of solutions of equations in Banach space, Trans. Amer. Math. Soc. (69): 208-231, 1950. MR0040578.

[Cr2] Cronin J. Fixed Points and Topological Degree in Nonlinear Analysis, American Mathematical Society, Providence, RI, 1964. MR0164101.

[Cr3] Cronin J. Differential Equations: Introduction and Qualitative Theory, Marcel Dekker, Inc., New York, 1980. MR0566401.

[Cr4] Cronin J. Mathematical Aspects of Hodgkin-Huxley Neural Theory, Cambridge University Press, Cambridge, 1987. MR0909892.

[Cr5] Cronin J. Electrically active cells and singular perturbation theory, Math. Intelligencer (12-4): 57-64, 1990. MR1076536.

[Cr6] Cronin J. Entrainment of frequency in singularly perturbed systems, Methods Appl. Anal. (3): 370-400, 1996. MR1421477.

[CM] Cronin J, O'Malley R (eds.). Analyzing Multiscale Phenomena Using Singular Perturbation Methods, Proceedings of Symposia in Applied Mathematics, 56, 1999. MR1718889.

[CS1] Cronin-Scanlon J. Advanced Calculus, D. C. Heath and Co., Boston, 1967. MR0217227.

[CS2] Cronin-Scanlon J. Letter to the Editor, Association for
Women in Mathematics (AWM) Newsletter, February 1972.

[CS3] Cronin-Scanlon J. Mathematical aspects of periodic catatonic schizophrenia, Bull. Math. Biology (39): 187199, 1977. MR0484501.

[ET] Ermentrout G, Terman D. Mathematical Foundations of Neuroscience, Springer-Verlag, 2010. MR2674516.

[GHW] Guckenheimer J, Hoffman K, Weckesser W, The Forced van der Pol Equation I: The Slow Flow and Its Bifurcations, SIAM J. Appl. Dynam. Sys. (2): 1-35, 2003. MR1975270.

[Le] Lefschetz L, review of Fixed Points and Topological Degree in Nonlinear Analysis, by Jane Cronin (MR0164101), Mathematical Reviews.

[LS] Leray J, Schauder J, Topologie et équations fonctionnelles, Ann. Sci. École Norm. Sup. (51): 45-78, 1934. MR1509338.

[Mu] Murray MAM, Women Becoming Mathematicians: Creating a Professional Identity in Post-World War II America, MIT Press, Cambridge, Mass./London, 2000. MR1778333.

[No] The Nobel Prize in Physiology or Medicine 1963. Nobel Media AB. Available at https://www. nobelprize.org /prizes/medicine/1963/summary. Accessed May 20, 2019.

[SI] Sleeman B, review of Mathematical Aspects of Hodgkin-Huxley Neural Theory, by Jane Cronin (MR909892, Mathematical Reviews.

[We1] Weibel C, A History of Mathematics at Rutgers. Available at https://www.math.rutgers.edu/for-alumni -friends/a-history-of-mathematics-at-rutgers. Accessed May 20, 2019.

[We2] Weibel C, Women in Rutgers Mathematics. Available at http://sites.math.rutgers.edu/ weibe1/Women-RU math.htm1. Accessed May 20, 2019.

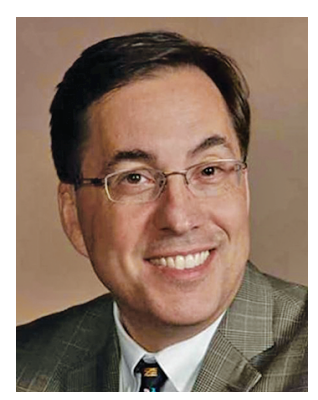

Edward F. Aboufadel

\section{Credits}

Figures 1 and 2 were created by the author using Mathematica.

Photos of Jane Scanlon are courtesy of Anne Scanlon Yamakaitis.

Author photo is courtesy of the author. 